\title{
Core-Polarization Contribution to the Nuclear Anapole Moment
}

\author{
N. Auerbach ${ }^{(1)}$ and B. A. Brown ${ }^{(2)}$ \\ (1) Raymond and Beverly Sackler Faculty of Exact Sciences, School of Physics and Astronomy, \\ Tel Aviv University, Ramat Aviv, Tel Aviv 69978, Israel \\ (2) NSCL/Cyclotron Laboratory and Department of Physics and Astronomy, Michigan State \\ University, East Lansing, MI 48824-1321, USA
}

(April 4, 2018)

\begin{abstract}
The importance of core contributions to the anapole moment in nuclei is examined. A model of the core-polarization correction is presented. The model is based on the coupling of the valence particles to the spin-dipole $J=$ $1^{-}$giant resonances of the core. A shell-model calculation of this correction is presented. The single-particle moments are calculated with Woods-Saxon and Skyrme Hartree Fock radial wave functions, and the general issues associated with nuclear configuration mixing are discussed.
\end{abstract}




\section{INTRODUCTION}

The recent observation of the anapole moment in ${ }^{133} \mathrm{Cs}[1]$, has spurred considerable interest in this subject. As remarked [2], this is the first observation of a static moment that is due to the violation of reflection symmetry. The existence of the anapole moment was suggested by Vaks and Zeldovich [3] early after the discovery of parity violation in $\beta$-decay.

The anapole moment exists in the situation when parity is violated but time reversal is preserved. Pioneering calculations of the anapole moment were done in ref. [4]. It was suggested that the anapole moment could provide information about the nature of the nucleon-nucleon $(\mathrm{N}-\mathrm{N})$ parity violating force, in particular about the $\pi$ and $\rho$ exchange contribution to the weak $\mathrm{N}-\mathrm{N}$ interaction. One of the immediate applications of the recent ${ }^{133} \mathrm{Cs}$ measurement was the attempt to try and deduce the pion-nucleon weak coupling constant $f_{\pi}[2],[5]$, by comparing the value of the measured anapole moment with the one calculated using a pure single-particle model. This comparison leads to a value for $f_{\pi}$ that exceeded by a factor 4 the value deduced from a hadronic parity violating measurement in ${ }^{18} F[6]$. At the present this controversy still exists. In this paper we wish to examine the role of core polarization and calculate its contribution to the values of the anapole moment. Some work in this respect has been done in the work of ref. [7]. Our calculations are analogous to the calculation of effective charges in nuclei, a concept well rooted in the field of nuclear structure.

The anapole operator is given by [1], [8]

$$
\hat{a}=-\pi \int d \vec{r} r^{2} \vec{j}(\vec{r})
$$

where $\vec{j}(\vec{r})$ is the nuclear electromagnetic current density. It has been found $[4,7,8]$ that the dominant part of the anapole operator stems from the spin part of $\vec{j}(\vec{r})$ and is given by:

$$
\hat{a}_{s}=\frac{\pi e \mu}{m}(\vec{r} \times \vec{\sigma})
$$

where $\mu$ is the nucleon magnetic moment, $m$ the nucleon mass, $\vec{\sigma}$ is the nucleon spin operator and $\vec{r}$ is its coordinate. 
For the nucleus we write this part of the anapole operator as

$$
\hat{a}_{s}=\frac{\pi e}{m} \sum_{i=1}^{A}\left[\mu+\left(\mu_{p}-\mu_{n}\right) t_{Z}(i)\right]\left(\vec{r}_{i} \times \vec{\sigma}_{i}\right)
$$

where $\mu=\frac{\mu_{p}+\mu_{n}}{2}, \mu_{p}, \mu_{n}$ are the proton and neutron magnetic moments in units of nuclear magnitons, $t_{Z}$ is the $\mathrm{Z}$ - component of the isospin operator, $\left(t_{Z}\right.$ for a proton is $+\frac{1}{2}$, and for a neutron $\left.-\frac{1}{2}\right)$. The sum $i$ is over all nucleons in the nucleus. The operator written in vector spherical harmonics and in terms of a tensor coupled product is:

$$
\vec{r}_{i} \times \vec{\sigma}_{i}=-i \sqrt{2} \sqrt{\frac{4 \pi}{3}} r_{i}\left[Y_{L=1}\left(\hat{r}_{i}\right) \otimes \vec{\sigma}\right]^{\Delta J=1}
$$

which is the $\Delta J^{\pi}=1^{-}, \mathrm{L}=1, \mathrm{~S}=1$ spin-dipole operator [9]. The anapole operator in eq. (3) involves therefore, the isoscalar and isovector $J=1^{-}$spin-dipole operators.

The distribution of isovector spin-dipole strength was studied extensively both experimentally and theoretically [10] in the eighties and there is a considerable amount of information about the isovector spin-dipole strength distribution. For the isoscalar spin-dipole there is little information.

The anapole moment is defined as the expectation value of $\hat{a}$.

$$
a=<\psi|\hat{a}| \psi>_{\left(J_{z}=J\right)}
$$

where $\psi$ is the ground state wave function of the nucleus. It is clear that since the operator $\hat{a}$ is odd under parity operation and time reversal operations (P-odd, T-odd) for a spin non-zero nuclear state - $a$ will be non-zero only if parity mixing occurs in the wave function $\psi$. [Note that one does not need time reversal violation in this case because, the operator $\hat{a}$ contains, unlike for example the electric dipole, the spin operator $\vec{\sigma}$. The anapole moment therefore is $\mathrm{P}$-odd and $\mathrm{T}$-even].

\section{GENERAL FORMALISM}




\section{A. The Single-Particle Contribution}

Let us consider a nucleus with a particle occupying an orbit $j_{+}$with positive parity. (The consideration that follow can be made by starting with a negative parity state $j_{-}$and interchanging simply the + and - indices). The ground state in first approximation can be written as:

$$
\left|\phi_{+}>=\right| 0^{+} j_{+}>
$$

$\left(0^{+}\right.$denotes the ground state spin of the core). Consider now a negative parity orbit with the same spin $j$ but opposite parity and lying above the $j_{+}$orbit. We denote this orbit as $j_{-}$.

$$
\left|\phi_{-}>=\right| 0^{+} j_{-}>
$$

In general the negative parity $j_{-}$orbit will be energetically about $1 \hbar \omega$ above $j_{+}$. A parity violating force will mix the two and we will have:

$$
\left|\phi_{+}^{\prime}>=\right| 0^{+} j_{+}>_{j}+\eta_{0} \mid 0^{+} j_{-}>_{j}
$$

with

$$
\eta_{0}=\frac{<\phi_{+}|W| \phi_{-}>}{\epsilon_{-}-\epsilon_{+}}
$$

Where $W$ is the parity violating interaction and $\epsilon_{+}, \epsilon_{-}$are the single-particle energies. We should remark here that $W$ is the effective parity violating interaction and may include also some many-body contributions, such as the excitation of the $0^{-}$spin-dipole [11]. The anapole moment from this admixture is:

$$
a_{s p}^{(p a r t)}=<\phi_{+}^{\prime}|\hat{a}| \phi_{+}^{\prime}>=2 \eta_{0}<0^{+} j_{+}|\hat{a}| 0^{+} j_{-}>
$$

In addition to the single-particle contribution involving the $j_{-}$orbit that is above the given $j_{+}$orbit, there is the equally important contribution of the orbit with spin $j$ equal to $j_{+}$but of negative parity lying $1 \hbar \omega$ below the orbit $j_{+}$. We denote this orbit as $j_{-}^{-1}$, 
indicating that it is a hole state. The ground state configuration $\mid 0^{+} j_{+}>$will mix with the 2p-1h configuration $\mid 0^{+} j_{+}^{2} j_{-}^{-1}>$.

$$
\left|\tilde{\phi}_{+}^{\prime}>=\right| 0^{+} j_{+}>_{j}+\tilde{\eta}_{0} \mid 0^{+} j_{+}^{2} j_{-}^{-1}>
$$

The contribution to the anapole of this mixing, we denote as $a_{s p}^{(h o l e)}$ and it is:

$$
a_{s p}^{(\text {hole })}=<\tilde{\phi}_{+}^{\prime}|\hat{a}| \tilde{\phi}_{+}^{\prime}>=2 \tilde{\eta}_{0}<0^{+} j_{+}|\hat{a}| 0^{+} j_{+}^{2} j_{-}^{-1}>
$$

As mentioned, the contribution of this term is of the same magnitude as $a_{s p}^{(\text {part) }}$. The sum of the two:

$$
a_{s p}=a_{s p}^{(p a r t)}+a_{s p}^{(h o l e)}
$$

will be considered here to represent the single-particle contribution. Let us now advance a bit and include in the wave function configurations involving excitation of the core.

\section{B. The Core-Polarization Model}

Of the many possible types of core excitations let us single out the components $\mid 1^{-} \otimes j_{+}^{\prime}>$ and $\mid 1^{-} \otimes j_{-}^{\prime}>$ involving single-particle states of positive and negative parity coupled to the spin-dipole resonances (isoscalar and isovector) to give total spin $j$. The symbol $\otimes$ denotes, angular momentum coupling. We will from now on not write this symbol in order to simplify notations. Now we have

$$
\left|\psi_{+}>=\alpha\right| 0^{+} j_{+}>_{j}+\sum_{j_{-}^{\prime}} \beta_{j^{\prime}-} \mid 1^{-} j_{-}^{\prime}>_{j}
$$

and

$$
\left|\psi_{-}>=\bar{\alpha}\right| 0^{+} j_{-}>_{j}+\sum_{j_{+}^{\prime}} \bar{\beta}_{j^{\prime}+} \mid 1^{-} j_{+}^{\prime}>_{j}
$$

In the following we will drop the index $j$ under the kets. For the sake of simplicity of our

presentation let us limit to one orbit $j^{\prime}$ taking $j_{-}^{\prime}=j_{-}$and $j_{+}^{\prime}$ being the next higher positive 
parity orbit after $j_{+}$. An extension to many orbits $j^{\prime}$ is immediate but complicates matters and notations. We consider therefore:

$$
\begin{gathered}
\left|\psi_{+}>=\alpha\right| 0^{+} j_{+}>+\beta \mid 1^{-} j_{-}> \\
\left|\psi_{-}>=\bar{\alpha}\right| 0^{+} j_{-}>+\bar{\beta} \mid 1^{-} j_{+}^{\prime}> \\
\beta=\frac{<0^{+} j_{+}\left|V_{N}\right| 1^{-} j_{-}>}{\Delta E_{\beta}} \\
\bar{\beta}=\frac{<0^{+} j_{-}\left|V_{N}\right| 1^{-} j_{+}^{\prime}>}{\Delta E_{\bar{\beta}}}
\end{gathered}
$$

where $V_{N}$ is the nuclear interaction.

The $W$ interaction will mix these two states and the parity mixed ground state will be:

$$
\left|\tilde{\psi}_{+}>=\right| \psi_{+}>+\eta \mid \psi_{-}>
$$

with

$$
\eta=\frac{<\psi_{+}|W| \psi_{-}>}{E_{-}-E_{+}}
$$

Since we expect $\alpha \gg \beta$ we can take $\eta \simeq \eta_{0}$. We evaluate $<\tilde{\psi}_{+}|\hat{a}| \tilde{\psi}_{+}>$(dropping the terms quadratic in $\eta_{0}$ ), and take $\alpha \simeq \bar{\alpha}=1$. We find

$$
a=2 \eta_{0}\left[<0^{+} j_{+}|\hat{a}| 0^{+} j_{-}>+\beta<0^{+} j_{-}|\hat{a}| 1^{-} j_{-}>+\bar{\beta}<1^{-} j_{+}^{\prime}|\hat{a}| 0^{+} j_{+}>\right]
$$

At this point we should note that because $\hat{a}$ is a one-body operator, the term involving $\bar{\beta}$ will be zero unless $j_{+}^{\prime}=j_{+}$. In this case $\left|\psi_{-}>=\bar{\alpha}\right| 0^{+} j_{-}>+\bar{\beta} \mid 1^{-} j_{+}>$. Then the two configurations $\mid 0^{+} j_{-}>$and $\mid 1^{-} j_{+}>$might be close in energy (if the spin-dipole is close to its unperturbed position). The contribution of this state will be large because of a large $\bar{\beta}$, however, its contribution will be cancelled by the nearby orthogonal partner state: $\left|\psi_{-}^{\prime}>=\bar{\beta}\right| 0^{+} j_{-}>-\bar{\alpha} \mid 1^{-} j_{+}>$.

Therefore: 


$$
a=2 \eta_{0}<0^{+} j_{+}|\hat{a}| 0^{+} j_{-}>\times\left[1+\frac{\beta<0^{+} j_{-}|\hat{a}| 1^{-} j_{-}>}{<0^{+} j_{+}|\hat{a}| 0^{+} j_{-}>}\right] \equiv a_{0}[1+\chi]
$$

where $\chi$ is the core contribution to the anapole moment.

$$
\chi=\frac{\beta<0^{+} j_{-}\left|\sum \vec{r}_{i} \times \vec{\sigma}_{i}\right| 1^{-} j_{-}>}{<0^{+} j_{+}\left|\sum \vec{r}_{i} \times \vec{\sigma}_{i}\right| 0^{+} j_{-}>}
$$

\section{A Simple Estimate}

We now proceed with some simple estimates, treating only the isovector spin-dipole. First, $\left|\epsilon_{j+}-\epsilon_{j-}\right| \simeq \hbar \omega,\left(\hbar \omega=41 A^{-1 / 3} \mathrm{MeV}\right)$ and $E_{1^{-}}=1 \hbar \omega+\Delta V$, where $\Delta V$, is a collective shift due to the interaction energy of the $1 \mathrm{p}$ - 1 h states forming the spin-dipole. The denominator in the expression for $\beta$ is approximately equal therefore to $\Delta E_{\beta} \simeq 2 \hbar \omega+\Delta V$. One can rewrite the expression for $\chi$ as:

$$
\chi=\frac{<0^{+} j_{+}\left|V_{N}\right| 1^{-} j_{-}><1^{-} j_{-}\left|\sum \vec{r}_{i} \times \vec{\sigma}_{i}\right| 0^{+} j_{-}>}{<0^{+} j_{+}|\vec{r} \times \vec{\sigma}| 0^{+} j_{-}>(2 \hbar \omega+\Delta V)}
$$

Proceeding with approximations we may now limit the $\sum \vec{r}_{i} \times \vec{\sigma}_{i}$ in the matrix elements of the numerator to the core nucleons and write:

$$
\chi=\frac{<1^{-}\left|\sum \vec{r}_{i} \times \vec{\sigma}_{i}\right| 0^{+}>}{<j_{+}|\vec{r} \times \vec{\sigma}| j_{-}>} \cdot \frac{<0^{+} j_{+}\left|V_{N}\right| 1^{-} j_{-}>}{2 \hbar \omega+\Delta V}
$$

We first note that the ratio

$$
\left|\frac{<1^{-}\left|\sum \vec{r}_{i} \times \vec{\sigma}_{i}\right| 0^{+}>}{<j+|\vec{r} \times \vec{\sigma}| j_{-}>}\right| \simeq \sqrt{N}
$$

could be a quite large number depending on the collectivity of the spin-dipole giant resonance. The symbol $N$ stands here for the effective number of particles that contribute to the collective spin-dipole. This number could be a source of enhancement of the core contribution to $a$. Let us now estimate the other quantities appearing in eq. (22).

The values of the matrix elements $<0^{+} j_{+}\left|V_{N}\right| 1^{-} j_{-}>$, or $<0^{+} j_{-}\left|V_{N}\right| 1^{-} j_{+}^{\prime}>$ can be estimated from the value of the collective shift $\Delta V=<1^{-}\left|V_{N}\right| 1^{-}>$. (See for example the discussion of particle + core coupling models in ref. [12]). On the average the values 
of the above particle + core coupling matrix elements should be equal to $\frac{\Delta V}{\sqrt{N}}$ where $\sqrt{N}$ is again the number of particles active in the formation of the spin-dipole. We may write the estimate

$$
\chi=\frac{\Delta V}{2 \hbar \omega+\Delta V}
$$

denoting $\lambda=\hbar \omega / \Delta V$

$$
\chi=\frac{1}{2 \lambda+1}
$$

For a large collective shift $(\Delta V=\hbar \omega) \chi$ is large. In ref. [9] the $1^{-}$isovector spin-dipole is

found to be at an energy $2 \hbar \omega$, meaning that $\Delta V \simeq 1 \hbar \omega$. In this case $\chi \simeq \frac{1}{3}$. We should stress that we are dealing with small admixtures of the core states. The admixtures for $\mid 1^{-} j>$ that are implied here are less than $1 \%$. It is the factor $\sqrt{N}$ in the spin-dipole strength that makes the $\chi$ correction sizable. If the collectivity of the spin-dipole is not high, one will find $\chi$ to be small. Our estimates are crude and one must have a more precise evaluation of the contribution of the core. In the next section, we describe such calculations.

\section{SHELL-MODEL CALCULATIONS}

\section{A. Matrix Elements and Operators}

In this section we describe details of the single-particle and configuration mixed anapole moment calculations. The spin-current contribution to the anapole moment is given by

$$
a_{s}=<\psi\left|\hat{a}_{s}\right| \psi>_{\left(J_{z}=J\right)}=\left(\begin{array}{ccc}
J & 1 & J \\
-J & 0 & J
\end{array}\right)<\psi\left\|\hat{a}_{s}\right\| \psi>
$$

where $J$ is the nuclear spin, () is the three-j symbol, and we use reduced matrix element convention of Edmonds [14]. In units where $\hbar=c=1$, the operator $\hat{a}_{s}$ is given by

$$
\hat{a}_{s}=\frac{\pi e}{m} \sum_{i=1}^{A} \mu_{i}\left(\vec{r}_{i} \times \vec{\sigma}_{i}\right)=-\frac{i \sqrt{2} \pi e}{m} \sqrt{\frac{4 \pi}{3}} \hat{a}_{s}^{\prime},
$$


where

$$
\hat{a}_{s}^{\prime}=\sum_{i=1}^{A} \mu_{i} r_{i}\left[Y_{L=1}\left(\hat{r}_{i}\right) \otimes \vec{\sigma}_{i}\right]^{\Delta J=1},
$$

and where $\mu_{i}$ are the nucleon magnetic moments in units of nuclear magnetons; $\mu_{p}=2.79$ and $\mu_{n}=-1.91$.

It is conventional to relate the anapole moment to a dimensionless constant $\kappa_{s}$ defined by:

$$
a_{s}=\frac{1}{e} \frac{G}{\sqrt{2}} \frac{K \kappa_{s}}{J(J+1)}<\psi|\vec{J}| \psi>_{\left(J_{z}=J\right)}=\frac{1}{e} \frac{G}{\sqrt{2}} \frac{K \kappa_{s}}{(J+1)}
$$

where

$$
K=\left(J+\frac{1}{2}\right)(-1)^{\ell+\frac{1}{2}-j}
$$

The $\ell$ and $j$ in the phase factor are chosen to be those of the dominate single-particle orbital associated with $\psi$.

The perturbation expansion of the reduced matrix element gives:

$$
<\psi\left\|\hat{a}_{s}\right\| \psi>=-2 \sum_{f} \frac{<\psi\left\|\hat{a}_{s}\right\| \phi_{f}><\phi_{f}|\hat{W}| \psi>}{|\Delta E|}
$$

where $\hat{W}$ is the weak interaction. For the weak interaction we use the approximation of Eq. (7) of [13]:

$$
\hat{W}=-\frac{i}{m} \frac{G}{\sqrt{2}} \sum_{i=1}^{A} g_{i} \frac{\vec{\sigma}_{i}}{2} \cdot\left[\vec{\nabla}_{i} \rho+\rho \vec{\nabla}_{i}\right]=-\frac{i}{m} \frac{G}{\sqrt{2}} \hat{W}^{\prime}
$$

with

$$
\hat{W}^{\prime}=\sum_{i=1}^{A} g_{i} \frac{\vec{\sigma}_{i}}{2} \cdot\left[\vec{\nabla}_{i} \rho+\rho \vec{\nabla}_{i}\right]
$$

where $g_{p}$ and $g_{n}$ are dimensionless constants representing the weak-interaction strength between the valence protons and neutrons, respectively, and the nuclear-matter density $\rho$. The nuclear-matter density is normalized by:

$$
\int \rho(r) d \vec{r}=A
$$


Finally, we express the dimensionless constant $\kappa_{s}$ in terms of the matrix elements of $\hat{a}_{s}^{\prime}$ and $\hat{W}^{\prime}$ :

$$
\kappa_{s}=\frac{\pi e^{2}}{m^{2}} \frac{\sqrt{2}(J+1)}{K}\left(\begin{array}{ccc}
J & 1 & J \\
-J & 0 & J
\end{array}\right) \sqrt{\frac{4 \pi}{3}} 2 \sum_{f} \frac{\left.<\psi\left\|\hat{a}_{s}^{\prime}\right\| \phi_{f}><\phi_{f}\left|\hat{W}^{\prime}\right| \psi\right\rangle}{|\Delta E|}
$$

Introducing the units of $\hbar$ and $c$, the constant in front becomes $\frac{\pi e^{2} \hbar^{2} c^{2}}{m^{2} c^{4}}=0.199 \mathrm{MeV} \mathrm{fm}^{3}$. The dimension of the $\hat{a}_{s}^{\prime}$ matrix element is fm and the dimension of the $\hat{W}^{\prime}$ matrix element is $\mathrm{MeV}^{-1} \mathrm{fm}^{-4}$.

In terms of the weak interaction, $\kappa_{s}$ is a linear combination of the single-particle coupling constants $g_{p}$ and $g_{n}$ which we will write as

$$
\kappa_{s}=g_{p} \kappa_{s p}+g_{n} \kappa_{s n}
$$

The total value of $\kappa_{s}$ will be obtained using the values [13] $g_{p}=4.5$ and $g_{n}=0$ based upon the DDH "best value" estimates [15]. The ultimate goal of comparing these type of calculations to experimental values for $\kappa$ will be to extract empirical values for $g_{p}$ and $g_{n}$ and use these to understand the nucleon-nucleon PNC weak interaction. The total value of $\kappa$ also involves the smaller convection-current contribution (see Eq. 67 of [13]). In this paper we focus only on the nuclear structure properties of the most important spin-current term $\kappa_{s}$.

\section{B. Single-Particle Terms}

First we consider the single-particle contributions to the intermediate states in Eq. 39. The cases we consider are those given in Table 3 of [13]. Specifically we start with closed shells for ${ }^{132} \mathrm{Sn}$ and ${ }^{208} \mathrm{~Pb}$. For ${ }^{133} \mathrm{Cs}$ we take a valence $1 \mathrm{~g}_{7 / 2}$ proton particle relative to ${ }^{132} \mathrm{Sn}$, for ${ }^{203,205} \mathrm{Tl}$ we take a $3 \mathrm{~s}_{1 / 2}$ proton hole relative to ${ }^{208} \mathrm{~Pb}$, for ${ }^{207} \mathrm{~Pb}$ we take a $3 \mathrm{p}_{1 / 2}$ neutron hole relative to ${ }^{208} \mathrm{~Pb}$ and for ${ }^{209} \mathrm{Bi}$ we take a $1 \mathrm{~h}_{9 / 2}$ proton particle relative to ${ }^{208} \mathrm{~Pb}$. We will discuss here the results based upon densities and radial wave functions in the matrix elements of $\hat{a}_{s}^{\prime}$ and $W^{\prime}$ which were obtained from Hartree-Fock (HF) calculations based on 
the SKX Skyrme interaction of [16]. The HF results will be compared to those from the Woods-Saxon (WS) potential of [17] for ${ }^{208} \mathrm{~Pb}$, and the interpolated parameter set of [18] for ${ }^{132} \mathrm{Sn}$.

In Eq. 39 we sum over all single-particle states $\phi$. The $\Delta E$ is the single-particle energy difference. This sum includes two type of terms: (1) the "hole" term in which the nucleons in occupied states are excited up to orbit $\psi$, e.g. $1 \mathrm{p}_{1 / 2}$ and $2 \mathrm{p}_{1 / 2}$ to $3 \mathrm{~s}_{1 / 2}$ for ${ }^{207} \mathrm{Tl}$, and (2), the "particle" term in which the nucleon in the orbit $\psi$ is excited into unoccupied states $\phi$, e.g. $3 \mathrm{~s}_{1 / 2}$ to $n \mathrm{p}_{1 / 2}$ with $n \geq 3$ for ${ }^{207} \mathrm{Tl}$. In the oscillator limit the $\vec{r}$ matrix element is nonzero only for the intermediate states which are $1 \hbar \omega$ away; e.g. $2 \mathrm{p}_{1 / 2}$ and $3 \mathrm{p}_{1 / 2}$ for ${ }^{207} \mathrm{Tl}$, and we find that with the more realistic HF and Woods-Saxon radial wave functions, that these " $1 \hbar \omega "$ terms are the only important ones. In cases where the unoccupied states are loosely bound or unbound, their radial wave functions are calculated by adding an external square well potential with a radius of $14 \mathrm{fm}$ and a depth of $20 \mathrm{MeV}$ to the HF potential. This has a negligible effect on the HF solution, but gives the unbound states a realistic excitation energy as well as an exponential fall off at large distance which is similar to the bound state $\psi$. The results are not sensitive to the exact values for the depth and radius of the external potential as long as it is sufficiently deep to bind the orbits and sufficiently large to not affect the HF bound state solution. The many-body aspect of the problem introduces an extra phase factor of -1 for the "hole" term in Eq. 39.

The HF and WS results are given in Table I. The WS results for the particle plus hole contributions are very close to the WS results given by Dmitriev et al. [13]. Furthermore, the HF results are very similar to WS. Most of the difference between HF and WS is due to the difference in the single-particle energy denominator of Eq. 39.

\section{Core-Polarization Correction}

Next we consider the admixture of particle-hole states in ${ }^{208} \mathrm{~Pb}$. The calculation is based

on the model space shown in Fig. 1 of [19] which for the ${ }^{208} \mathrm{~Pb}$ closed shell has $1 \mathrm{~g}_{7 / 2}, 2 \mathrm{~d}_{5 / 2}$, 
$2 \mathrm{~d}_{3 / 2}, 3 \mathrm{~s}_{1 / 2}$ and $1 \mathrm{~h}_{11 / 2}$ filled orbits for protons, $1 \mathrm{~h}_{9 / 2}, 2 \mathrm{f}_{7 / 2}, 2 \mathrm{f}_{5 / 2}, 3 \mathrm{p}_{3 / 2}, 3 \mathrm{p}_{1 / 2}$ and $1 \mathrm{i}_{13 / 2}$ empty orbits for protons, $1 \mathrm{~h}_{9 / 2}, 2 \mathrm{f}_{7 / 2}, 2 \mathrm{f}_{5 / 2}, 3 \mathrm{p}_{3 / 2}, 3 \mathrm{p}_{1 / 2}$ and $1 \mathrm{i}_{13 / 2}$ filled orbits for neutrons and $1 \mathrm{i}_{11 / 2}, 2 \mathrm{~g}_{9 / 2}, 2 \mathrm{~g}_{7 / 2}, 3 \mathrm{~d}_{3 / 2}, 4 \mathrm{~s}_{1 / 2}, 2 \mathrm{~g}_{7 / 2}, 3 \mathrm{~d}_{5 / 2}$ and $1 \mathrm{j}_{15 / 2}$ empty orbits for neutrons. We note that this model space includes the necessary orbits for the "particle" admixtures in ${ }^{207} \mathrm{Th}$ and ${ }^{207} \mathrm{~Pb}$ and thus we will focus our calculations of the spin-dipole correlation effects on these two nuclei.

The Hosaka G matrix [20] was used for the residual strong interaction, and the singleparticle energies were fixed to reproduce the experimental single-particle energies as given in Fig. 1 of [19]. The use of the Hosaka G matrix for the ${ }^{208} \mathrm{~Pb}$ and its comparison with other G matrix interactions is discussed in [19].

For the $1^{-}$states of interest here there are 27 particle-hole configurations. One of these is spurious and it was removed from the spectrum by using the method of Glockner and Lawson [21] of applying a center-of-mass hamiltonian to raise the energy of the spurious state and remove its effect from the low-lying states of interest. (Even though this is a large model space, there are six dipole excitations missing space, e.g. $1 \mathrm{~h}_{11 / 2}$ to $1 \mathrm{i}_{11 / 2}$ for protons.) We show in Fig. 1, the single-particle dipole and spin-dipole response for transitions to $1^{-}$states in ${ }^{208} \mathrm{~Pb}$. Specifically, the spin-dipole strength $B(a)$ is given by the reduced matrix element of $\hat{a}_{s}^{\prime}$. The unperturbed results shown in Fig. 1 were obtained using the single-particle energies of [19] and with the center-of-mass hamiltonian, but with no residual interaction. When the Hosaka G matrix is used, the dipole strength moves from its unperturbed position of $7-8 \mathrm{MeV}$ to to a collective state at $11.7 \mathrm{MeV}$. The experimental giant dipole in ${ }^{208} \mathrm{~Pb}$ [12] lies at about $13.5 \mathrm{MeV}$ with an energy weighted sum-rule strength of about $100 \%$ of the classical sum-rule value of $14.8 \mathrm{NZ} / A=735 \mathrm{e}^{2} \mathrm{fm}^{2} \mathrm{MeV}$. The total experimental $\mathrm{B}(\mathrm{E} 1)$ strength is thus about $54 \mathrm{e}^{2} \mathrm{fm}^{4}$ and the total one-particle one-hole (1p-1h) strength in our calculation is $88 \mathrm{e}^{2} \mathrm{fm}^{4}$. The results for the dipole and spin-dipole strength functions are shown in Fig. 2. We note that the spin-dipole strength is collective and is pushed up in energy compared to the single-particle limit of Fig. 1, but it is not as collective as the isovector dipole. The levels for the low-lying mixed $1 \mathrm{p}-1 \mathrm{~h}$ states in ${ }^{208} \mathrm{~Pb}$ obtained with the 
G matrix interaction are in excellent agreement with experiment, within typically $130 \mathrm{keV}$ [22].

Next we recalculate the "particle" anapole matrix elements for ${ }^{207} \mathrm{Th}$ and ${ }^{207} \mathrm{~Pb}$ by using ground state wave which include mixing from the $1 \mathrm{p}-1 \mathrm{~h}$ states in ${ }^{208} \mathrm{~Pb}$. For example for ${ }^{207} \mathrm{Th}$, this admixture consists of the $3 \mathrm{p}_{1 / 2}$ particle coupled to all of the $1 \mathrm{p}-1 \mathrm{~h}$ states. Since this is a " $2 \hbar \omega$ " admixture the spurious center-of-mass motion can only be removed approximately. Its strength was chosen so that the mostly nonspurious admixed states were stabilized around 8-20 MeV excitation energy above the single-particle ground state, whereas the mostly spurious state was pushed to about $100 \mathrm{MeV}$ excitation energy. If the spurious state is not removed it comes at a very low excitation energy and mixes strongly with the single-particle ground state. On the other hand if the center-of-mass hamiltonian is too strong, all states are moved up too high (because of their small but non-zero spurious component) and these is no mixing of the non-spurious components of interest with the single-particle state.

The results are given in Table II next to the column labeled "HF part+CP". It turns out the the effect of the core-polarization is rather small, resulting in about a 10\% reduction for

${ }^{207} \mathrm{Th}$ and a $5 \%$ enhancement for ${ }^{207} \mathrm{~Pb}$. These core-polarization corrections arise from both the $\hat{a}_{s}^{\prime}$ and $\hat{W}^{\prime}$ matrix elements in Eq. 39, but the dominant effect is on the $\hat{a}_{s}^{\prime}$ term. The core-polarization admixtures in the ground states is only about $1 \%$, yet the effect is rather significant. These calculations indicate that the core-polarization is not too large, but not negligible. The calculations for the core-polarization contributions might be expanded in future work by using a perturbation approach.

\section{Configuration Mixing and Comparison to Experiment}

The final step for the anapole moment calculations will be to go from the "single-particle" nuclei around ${ }^{132} \mathrm{Sn}$ and ${ }^{208} \mathrm{~Pb}$ to the multi-valence-particle configurations involved in those nuclei where measurements have been carried out, in particular for ${ }^{133} \mathrm{Cs}$ and ${ }^{205} \mathrm{Th}$. The 
main complication here is that the anapole moment will consist of a linear combination of diagonal (e.g. $<\psi\|\hat{a}\| \psi>$ ) and off-diagonal (e.g. $<\psi\|\hat{a}\| \psi^{\prime}>$ ) reduced matrix elements within the valence space.

Good shell-model hamiltonians exist for ${ }^{205} \mathrm{Th}$ [23]. It is known in this case [23] that the diagonal matrix element for the $3 \mathrm{~s}_{1 / 2}$ orbit gets reduced from by about 0.80 compared to its value in ${ }^{207} \mathrm{Th}$ due to configuration mixing. We have use the HF wave functions to calculate all of the single-particle anapole matrix elements involved in the ${ }^{205} \mathrm{Th}$ ground state. When these are combined with the $\Delta J=1$ one-body transition densities for the ${ }^{205} \mathrm{Th}$ ground state, the anapole moment comes out be 0.40 ; close to the $3 \mathrm{~s}_{1 / 2}$ single-particle value. As mentioned, the diagonal matrix element is reduced, but the smaller off-diagonal terms give some enhancement which cancels out the reduction. The core-polarization correction considered above would reduce this to about 0.35 . To obtain the final value of the anapole moment we add the additional (smaller) term from the convection-current contribution as given in Table 3 of Dmitriev et al. [13] which is about -0.09 . The total calculated anapole moment is thus about 0.24 , which should be compared with the experiment value of $-0.22 \pm 0.30$ [24]. The agreement is fair given the large experimental error.

The single-particle value for the spin contribution to the anapole moment for ${ }^{133} \mathrm{Sb}$ from Table I is 0.29 which, when added to the convection-current contribution from Table 3 of Dmitriev (-0.05), gives a total anapole moment of 0.25 . This is in fair agreement with the results of $0.36 \pm 0.06$ deduced in [2] on the basis of atomic physics considerations from the experiment on ${ }^{133} \mathrm{Cs}$ atoms [1]. However, one cannot make a final comparison between theory and experiment until one has a calculation for the core-polarization contribution as well as for the structure of ${ }^{133} \mathrm{Cs}$. A hamiltonian for this mass region has been developed and applied to the magnetic moment of ${ }^{137} \mathrm{Cs}[25]$, where one finds that the diagonal $\lg _{7 / 2}$ term is within a few percent of its value in ${ }^{133} \mathrm{Sb}$. However, for ${ }^{133} \mathrm{Cs}$ the spherical dimensions involved in valence space are extremely large (on the order of $10^{9}$ ) and one will have to explore the use of the deformed single-particle model or the shell-model Monte Carlo method in order to carry out a reliable calculation. 


\section{CONCLUSIONS}

We have investigated anapole moments in heavy nuclei in the framework of shell-model configuration mixing. The single-particle anapole moments are broken down into their components coming from the weak-interaction mixing of particle and hole terms. The sum of these terms calculated with Woods-Saxon radial wave functions are close to the values obtained by Dmitriev et al. [13]. We have also evaluated these matrix elements with Skyrme Hartree-Fock radial wave functions and the results are similar to those obtained with the Woods-Saxon potential. We discuss the general principle behind the core-polarization corrections to the anapole moment. Specific calculations are carried out for ${ }^{208} \mathrm{~Pb}$ with a G matrix interaction which incorporates realistic collective states for the spin-dipole excitation. The core-polarization corrections for the "particle" contributions in ${ }^{207} \mathrm{Th}$ and ${ }^{207} \mathrm{~Pb}$ turn out to be on the order of $10 \%$; not very large, but not negligible. The single-particle matrix elements which we could consider in these calculations were, however, limited, and it would be desirable to carry out more extensive calculations along these lines. We have also made a configuration mixing calculation for the anapole moment of ${ }^{205} \mathrm{Th}$ which gave a value close to the previous single-particle estimate and in fair agreement with experiment.

Acknowledgements: This work was supported in part by the US-Israel Binational Science Foundation and by NSF grant PHY-9605207. 


\section{Figure Captions:}

Figure 1: $B(E 1)$ and $B(a)$ strength distributions in ${ }^{208} \mathrm{~Pb}$ obtained with the unperturbed $1 \mathrm{p}-1 \mathrm{~h}$ wave functions.

Figure 2: $B(E 1)$ and $B(a)$ strength distributions in ${ }^{208} \mathrm{~Pb}$ obtained with the mixed $1 \mathrm{p}-1 \mathrm{~h}$ wave functions. 


\section{REFERENCES}

[1] C.S. Wood et al., Science 275, 1759 (1997).

[2] V. V. Flambaum and D. W. Murray, Phys. Rev. C56, 1641 (1997).

[3] Ya. B. Zeldovich, Sov. Phys. JETP 6, 1184 (1958).

[4] V. V. Flambaum and I. B. Khriplovich, Sov. Phys. JETP 52, 835 (1990).

[5] W.C. Haxton, Science 275, 1753 (1997).

[6] E. G. Adelberger and W. C. Haxton, Annu. Rev. Nucl. Part. Sci 35 , 501 (1985).

[7] I. Khriplovich, Parity Nonconservation in Atomic Phenomena (Gordon Breach, Philadelphia 1991)

[8] V. F. Dmitriev and V. B. Telitsin, Nucl. Phys. A613, 237 (1997).

[9] N. Auerbach and A. Klein, Phys. Rev. C30, 1032 (1984).

[10] F. Osterfeld, Rev. of Mod. Phys. 64, 491 (1992), and references therein.

[11] N. Auerbach, Phys. Rev. C45, R514 (1992).

[12] A. Bohr and B. R. Mottelson, Nuclear Structure Vol. II, (W. A. Benjamin, New York, 1975).

[13] V.F. Dmitriev, J.B. Khriplovich and V.B. Telitsen. Nucl. Phys. A577, 691 (1994).

[14] A. R. Edmonds, Angular Momentum in Quantum Mechanics, (Princeton University Press, 1960).

[15] B. Desplanques, J. F. Donoghue and B. R. Holstein, Ann. Phys. 124, 449 (1980).

[16] B. A. Brown, Phys. Rev. C58, 220 (1998).

[17] B. A. Brown, S. E. Massen, J. I. Escudero, P. E. Hodgson, G. Madurga and J. Vinas, J. Phys. G9, 423 (1983). 
[18] J. Streets, B. A. Brown and P. E. Hodgson, J. Phys. G8, 839 (1982).

[19] E. K. Warburton and B. A. Brown, Phys. Rev. C43, 602 (1991).

[20] A. Hosaka, K. I. Kubo and H. Toki, Nucl. Phys. A244, 76 (1985).

[21] D. H. Glockner and R. D. Lawson, Phys. Lett. 53B, 313 (1974).

[22] M. Schramm et al., Phys. Rev. C56, 1320 (1997); M. Rejmund, Dissertation, Univ. of Warsaw, 1999.

[23] L. Rydstrom, J. Blomqvist, R. J. Liotta and C. Pomar, Nucl. Phys. A512, 217 (1990).

[24] P. A. Vetter, D. N. Meekhof, P. K. Majumder, S. K. Lamoreaux and E. N. Fortson, Phys. Rev. Lett. 74, 2658 (1995).

[25] G. N. White et al., Nucl. Phys. A644, 277 (1998). 


\section{TABLES}

TABLE I. Single-particle $\kappa_{s}$ values for the anapole moments.

\begin{tabular}{|c|c|c|c|c|c|c|}
\hline Nucleus & single-particle & contribution & $|\Delta E|(\mathrm{MeV}) \mid$ & $\kappa_{s p}$ & $\kappa_{s n}$ & $\kappa_{s}$ \\
\hline${ }^{207} \mathrm{Tl}$ & $3 s_{1 / 2}$ & $\begin{array}{c}\text { WS hole } \\
\text { WS part } \\
\text { WS hole+part } \\
\text { HF hole } \\
\text { HF part } \\
\text { HF hole+part }\end{array}$ & $\begin{array}{r}7.63 \\
7.89 \\
\\
8.32 \\
8.67\end{array}$ & $\begin{array}{l}0.042 \\
0.064 \\
0.106 \\
0.038 \\
0.058 \\
0.095\end{array}$ & & $\begin{array}{l}0.188 \\
0.287 \\
0.475 \\
0.169 \\
0.259 \\
0.429\end{array}$ \\
\hline${ }^{207} \mathrm{~Pb}$ & $3 p_{1 / 2}$ & $\begin{array}{c}\text { WS hole } \\
\text { WS part } \\
\text { WS hole+part } \\
\text { HF hole } \\
\text { HF part } \\
\text { HF hole+part }\end{array}$ & $\begin{array}{l}7.89 \\
6.29 \\
\\
7.83 \\
6.64\end{array}$ & & $\begin{array}{l}-0.044 \\
-0.046 \\
-0.090 \\
-0.044 \\
-0.044 \\
-0.088\end{array}$ & $\begin{array}{l}0.0 \\
0.0 \\
0.0 \\
0.0 \\
0.0 \\
0.0\end{array}$ \\
\hline${ }^{209} \mathrm{Bi}$ & $1 h_{9 / 2}$ & $\begin{array}{c}\text { WS hole } \\
\text { WS part } \\
\text { WS hole+part } \\
\text { HF hole } \\
\text { HF part } \\
\text { HF hole+part }\end{array}$ & $\begin{array}{l}11.44 \\
7.36 \\
\\
11.43 \\
9.10\end{array}$ & $\begin{array}{l}0.062 \\
0.024 \\
0.086 \\
0.063 \\
0.019 \\
0.079\end{array}$ & & $\begin{array}{l}0.281 \\
0.106 \\
0.386 \\
0.283 \\
0.073 \\
0.356\end{array}$ \\
\hline${ }^{133} \mathrm{Sb}$ & $1 g_{7 / 2}$ & $\begin{array}{c}\text { WS hole } \\
\text { WS part } \\
\text { WS hole+part } \\
\text { HF hole } \\
\text { HF part }\end{array}$ & $\begin{array}{l}12.47 \\
7.87 \\
\\
12.80 \\
9.62\end{array}$ & $\begin{array}{l}0.048 \\
0.023 \\
0.071 \\
0.047 \\
0.017\end{array}$ & & $\begin{array}{l}0.216 \\
0.103 \\
0.319 \\
0.210 \\
0.077\end{array}$ \\
\hline
\end{tabular}




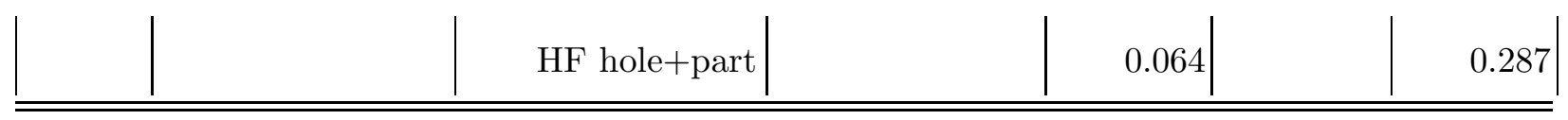

TABLE II. Core-polarization corrections for the anapole moments.

\begin{tabular}{|c|r|r|r|r|r|r|}
\hline \hline Nucleus & single-particle & contribution & $\begin{array}{r}|\Delta E| \\
(\mathrm{MeV})\end{array}$ & $\kappa_{s p}$ & $\kappa_{s n}$ & $\kappa_{s}$ \\
\hline${ }^{207} \mathrm{Tl}$ & $3 s_{1 / 2}$ & HF part & 8.67 & 0.058 & & 0.259 \\
& $3 p_{1 / 2}$ & HF part+CP & 8.67 & 0.053 & 0.004 & 0.239 \\
\hline${ }^{207} \mathrm{~Pb}$ & & HF part & 6.64 & & -0.044 & 0.0 \\
& & HF part+CP & 6.64 & -0.001 & -0.046 & -0.006 \\
\hline \hline
\end{tabular}




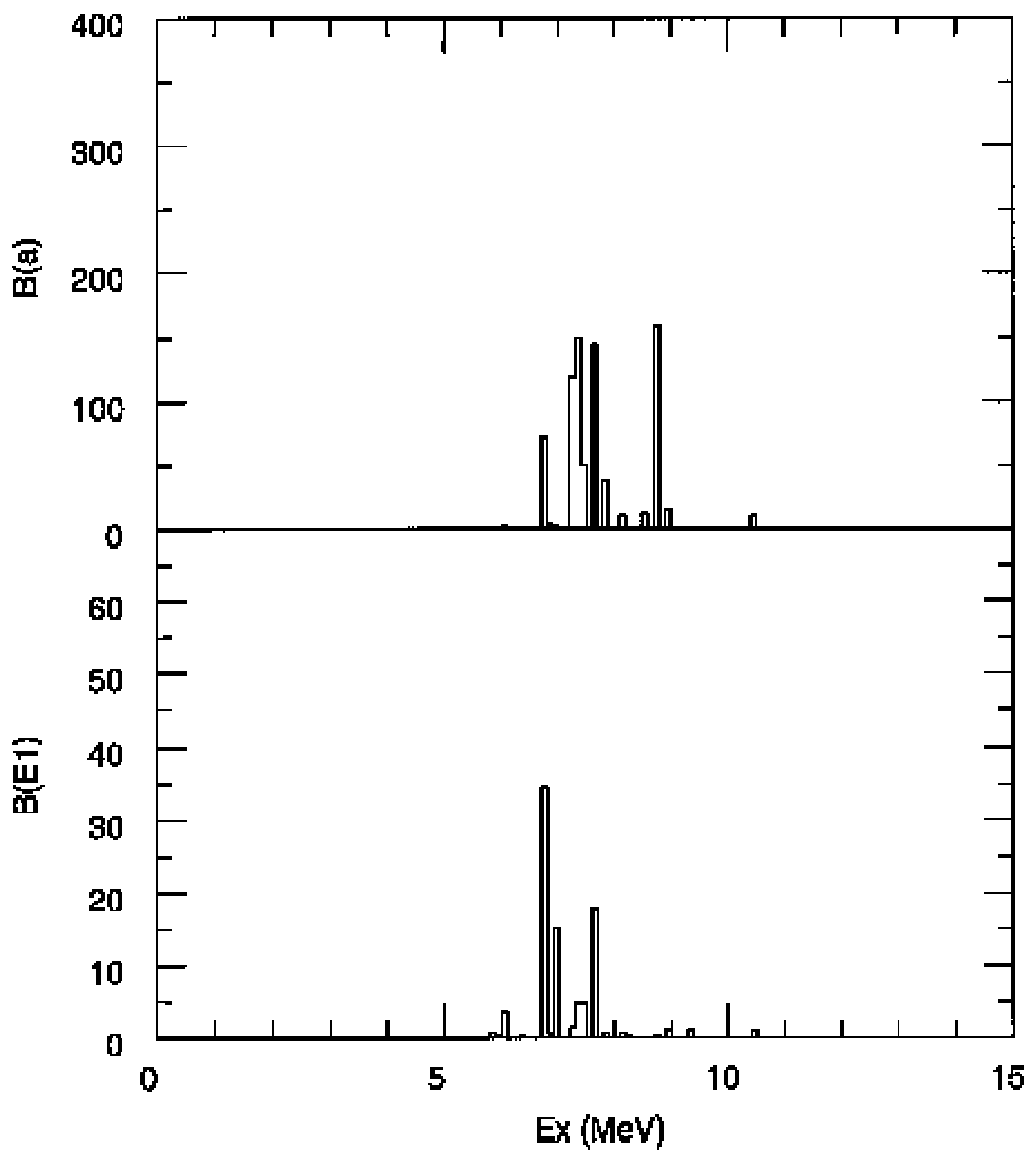

Flgure 1 


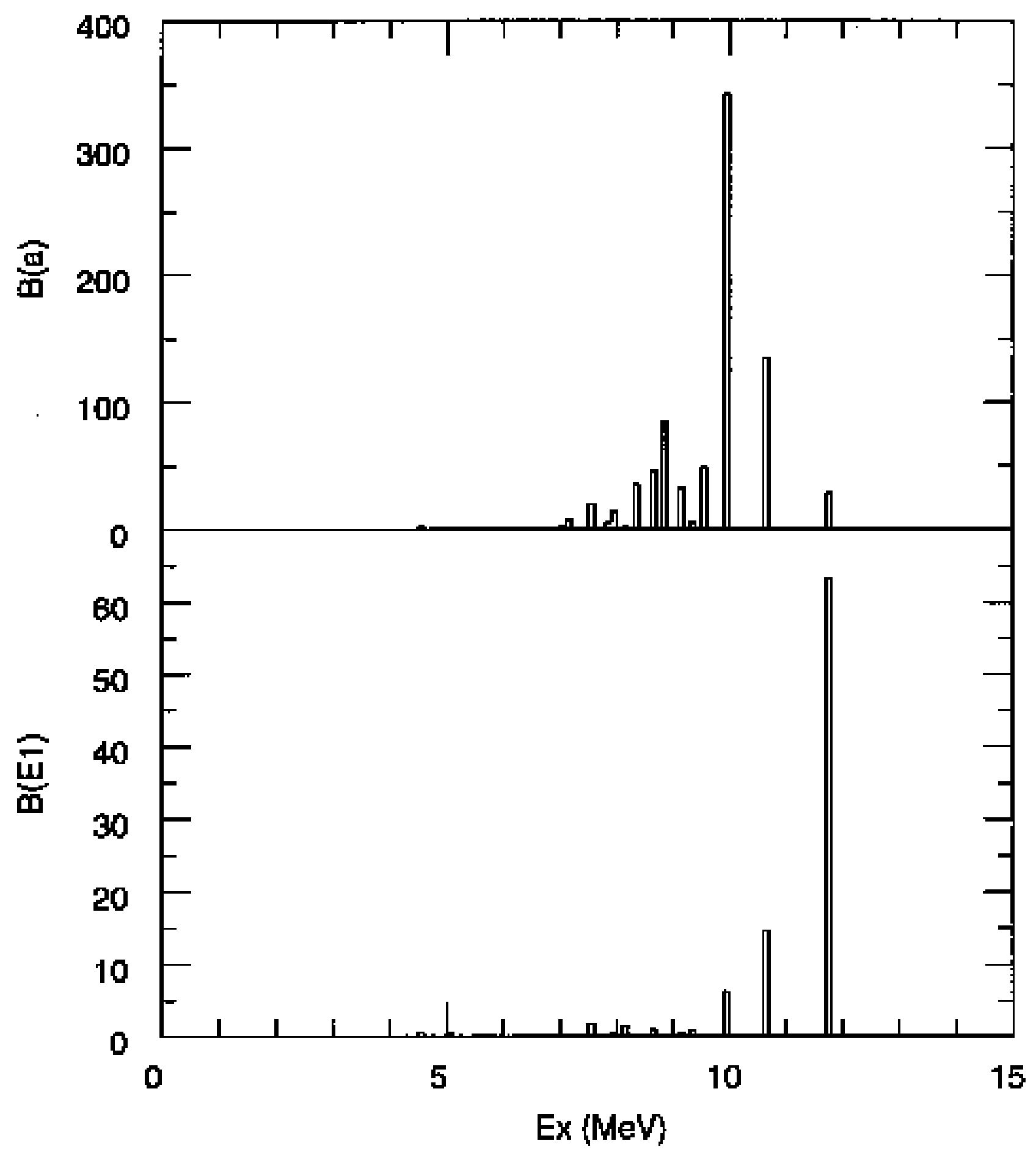

Figure 2 